\title{
European Association of Urology Position Statement on Screening for Prostate Cancer
}

\author{
Per-Anders Abrahamsson ${ }^{a, 1, *}$, Walter Artibani ${ }^{b, 1}$, Christopher R. Chapple ${ }^{c, 1}$, Manfred Wirth $^{d, 1}$ \\ a Department of Urology, Malmö University Hospital, Lund University, S-205 02 Malmö, Sweden \\ ${ }^{\mathrm{b}}$ Department of Oncological and Surgical Sciences, Urology Clinic, University of Padua, Padua, Italy \\ ${ }^{\mathrm{c}}$ Sheffield Teaching Hospitals NHS Trust, Urology Research, Royal Hallamshire Hospital, Glossop Road, Sheffield, South Yorkshire S10 2JF, \\ United Kingdom \\ ${ }^{\mathrm{d}}$ Carl Gustav Carus - Medical School, Technical University of Dresden, Department of Urology, Fetscherstr. 74, Dresden 01307, Germany
}

The European Association of Urology (EAU) has taken into consideration the recent scientific information on randomised screening studies on prostate cancer [1,2]. Based on the results of the European Randomised Study of Screening for Prostate Cancer (ERSPC) [1], the EAU has formulated a position statement regarding prostate cancer screening in Europe and the subsequent actions to be taken by health professionals and health authorities.

The ERSPC reports on a relative prostate cancer mortality reduction of at least $20 \%$ by prostate-specific antigen (PSA)-based population screening in 162,000 asymptomatic men aged 55-69 yr. For every prostate cancer death prevented, 1410 men have to undergo screening, while 48 need to be treated in excess of the control group population to save 1 prostate cancer death. Results of the randomised Prostate, Lung, Colorectal, and Ovarian (PLCO) Cancer Screening Trial in the United States [2] were published at the same time as the ERSPC results. To date, results from this study show no significant effect of screening on mortality from prostate cancer, but the study suffered from a significant level of contamination in the control arm. The study continues.

With these data in mind, the EAU has adopted the conclusions of the ERSPC study and recognises the benefit of screening in terms of mortality reduction, as well as the adverse effects of overdiagnosis and overtreatment of prostate cancers that could be quantified for the first time in the setting of a randomised screening study. Further publication of relevant data is awaited from the ERSPC group and will be used to inform the debate.

In the interest of men's health in Europe and elsewhere, the EAU has formulated the following statements:

- Prostate cancer is a major health problem and one of the main causes of male cancer death. However, current published data are insufficient to recommend the adoption of population screening for prostate cancer as a public health policy because of the significant overtreatment that would result. Before screening is considered by national health authorities, the level of current opportunistic screening as well as issues of overdiagnosis, overtreatment, quality of life, cost, and cost-effectiveness should be taken into account.

- Overdiagnosis of prostate cancer potentially leads to significant overtreatment of prostate cancer. Health professionals, especially urologists, should avoid overtreatment by developing safe methods of cancer surveillance and monitoring without resorting to invasive therapy. Invasive therapies should be tailored to patients' needs and the prognosis of cancers diagnosed.

- Current screening algorithms are insufficient because of their lack of specificity and lack of selectivity for aggressive cancers that require treatment. The development of novel diagnostic and prognostic markers and imaging modalities is urgently needed to enhance the predictive value of screening tools.

\footnotetext{
* Corresponding author. Tel. +46 40331000; Fax: +46 40336283.

E-mail address: Per-anders.Abrahamsson@skane.se (P.-A. Abrahamsson).

1 The EAU Executive Committee, 2009.
} 
- In the absence of population screening, the EAU advises men who consider screening by PSA testing and prostate biopsy to obtain information on the risks and benefits of screening and make an individual risk assessment.

- The EAU and the ERSPC study group represent essential European stakeholders who will further develop health strategies for prostate cancer screening.

- The EAU promotes the quality of care for prostate cancer patients in Europe in collaboration with the patient support organisation Europa Uomo through the development of information support and guidelines.

- The EAU wishes to support and foster research needed to develop reliable active-surveillance protocols for low-risk prostate cancers, prognostic markers, and targeted therapies to deliver optimal patient care.

Conflicts of interest: The authors have nothing to disclose.

\section{References}

[1] Schröder F, Hugosson J, Roobol M, et al. Screening and prostatecancer mortality in a randomized European study. N Engl J Med 2009;360:1320-8.

[2] Andriole G, Crawford D, Grubb R, et al. Mortality results from a randomized prostate-cancer screening trial. N Engl J Med 2009; 360:1310-9.

\section{Embracing Excellence in Prostate, Bladder}

\section{and Kidney Cancer}

\section{7-29 November 2009}

Barcelona, Spain

www.emucbarcelona2009.org

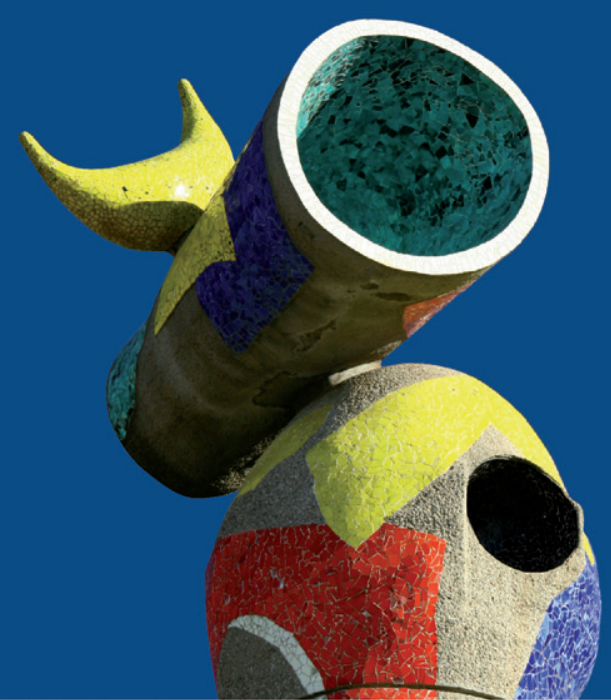

$2^{\text {nd }}$ European Multidisciplinary Meeting on Urological Cancers organised by: 